\title{
Improved Modelling of Ultrasound Contrast Agent Diminution for Blood Perfusion Analysis
}

\author{
Christian Kier ${ }^{1}$, Karsten Meyer-Wiethe ${ }^{2}$, Günter Seidel ${ }^{2}$, and Alfred Mertins ${ }^{1}$ \\ 1 Institute for Signal Processing, University of Lübeck, Germany \\ kier@isip.uni-luebeck.de \\ 2 Department of Neurology, University Medical Center Schleswig-Holstein, \\ Lübeck, Germany
}

\begin{abstract}
Ultrasound contrast imaging is increasingly used to analyze blood perfusion in cases of ischemic or cancerous diseases. Among other imaging methods, the diminution harmonic imaging (DHI), which modells the diminution of contrast agent due to ultrasound pulses, is the most promising because of its speed. However, the current imaging quality of DHI is insufficient for reliable diagnoses.

In this paper, we extend the mathematical DHI model to include the part of the intensity signal which is due to tissue reflections and other effects not based on the contrast agent and its concentration in the blood. We show in a phantom experiment with available perfusion ground truth the vast improvements in accuracy of the new model. Our findings also strongly support the theory of a linear relationship between the perfusion speed and the determined perfusion coefficient, which is a large step towards quantitative perfusion measurements.
\end{abstract}

\section{Introduction}

Ultrasound (US) perfusion analysis with ultrasound contrast agents (UCA) is a growing field. Its applications cover all major areas in the body, e.g. heart, liver, or brain 12. Due to the use of ultrasound it has several advantages over the use of CT/MRI-based methods, for instance reduced time and cost, and the applicability to critically ill patients as a bedside method. However, the imaging quality is not yet on the level accustomed by CT or MRI.

The most widely used imaging method is based on analyzing the kinetics of a UCA bolus. Studies have shown its diagnostic significance [3. Its main drawback however is its long duration, which also makes it prone to movement artifacts 4. Other methods are based on continuous UCA infusion, such as the diminution method. It uses high-power US pulses to destroy a substantial part of the contrast agent with each pulse. A steady state is reached after a couple of pulses, in which every pulse destroys as much UCA as is washed in during the interframe interval. Instead of covering a whole bolus, which takes up to $60 \mathrm{~s}$, the DHI method acquires only 6-10 images at an interframe interval of 150 $1000 \mathrm{~ms}$. The first approach to model the DHI method and extract perfusionrelated parameters appeared in [5], where the model was fitted to the data by 

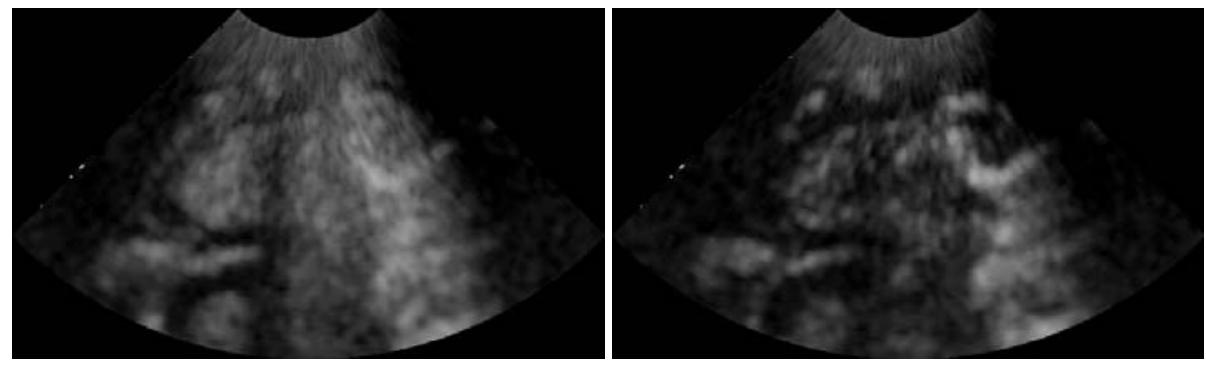

Fig. 1. First (left) and last (right) image of an exemplary transcranial DHI sequence

least squares methods. An improvement was made by [6] to be able to directly calculate the parameters and avoid the time-consuming fitting process. Other approaches tried to determine the perfusion in a general way, independent of the actual UCA concentration and insonation pattern used [7]. However, they do not seem to have found wide acceptance.

This paper is organized as follows: Section 2 explains the DHI method in detail and presents our model extensions and their implications. In Section 3 , the results of a phantom study are shown to prove the higher accuracy of the new model. Finally, in Section 4 the results are discussed and conclusions are drawn.

\section{Methods}

\subsection{Diminution Harmonic Imaging}

To improve the SNR, ultrasound harmonic imaging makes use of the fact that the ultrasound attenuation varies over frequency. The use of UCA amplifies this effect. UCA consist mainly of gas-filled microbubbles that nonlinearly scatter ultrasound and hence improve harmonic imaging. Depending on the US pulse energy, the microbubbles are even destroyed, which also generates a nonlinear echo. While the US pulse is sent with a center frequency of $1.8 \mathrm{MHz}$, the probe records also harmonic frequencies which are mainly due to contrast agent. Since contrast agent can only be found in the blood circulation, this procedure is well suited for perfusion imaging. Analyzing a varying contrast agent concentration in an US image sequence provides information about the actual perfusion in the imaging plane.

The DHI method is based on continuous contrast agent infusion leading to a constant UCA concentration in the blood. A series of US pulses reduces the UCA concentration, since microbubbles are destroyed due to the high sound pressure using this method. After approximately 5 pulses, the UCA concentration approaches an equilibrium, in which an US pulse destroys as much contrast agent as is flowing into the image plane during one interpulse interval. Fig. 1 shows the first and last image from an exemplary DHI sequence (in this case from the brain). The intensity decrease is clearly shown although in some high blood flow 
velocity regions, like the posterior cerebral artery, the intensity almost did not change.

\subsection{Mathematical Model}

Our model is specific to the DHI process, which we consider special even among ultrasound perfusion analysis models. As opposed to many other perfusion analysis approaches, the DHI process directly depends on the insonation pattern. Hence, the measurement itself drastically influences the measured process.

As shown in Fig. 1, in some regions the image intensity does not change very much or decreases only to a certain level. This is due to the fact that the US system receives not only UCA echos but also tissue signals. Although quite obvious, this baseline intensity $I_{b}$ was to our knowledge not yet modelled explicitly. Instead, its incorporation with the model was considered unnecessary [5].

Furthermore, the UCA concentration is modelled while only the image intensity $I(n)$ is observable. Although considered the most critical precondition, past publications did not make an assumption on the relationship between UCA concentration and image intensity, but used the intensity values directly. Due to equally distributed UCA and unchanged acoustic power and insonation plane, the remaining fraction of UCA $d$ is assumed to be constant. Hence, a linear relationship between the UCA concentration $C(n)$ and the UCA-dependent image intensity $I_{c}(n)$ can be assumed, which is supported by our experimental results:

$$
\begin{aligned}
I(n) & :=I_{c}(n)+I_{b} \\
I_{c}(n) & :=k \cdot C(n)
\end{aligned}
$$

where $n$ is the index for an insonation pulse, since the process can only be observed at discrete intervals. Three factors influence the UCA concentration $C(n)$ in the insonation plane: blood wash-in, wash-out, and UCA destruction by US pulses. Hence, it can be modelled as the following function in the sampled tissue/blood volume:

$$
C(n+1)=C(1) \cdot \underbrace{\left(1-e^{-p \cdot \Delta t}\right)}_{\text {Inflow }}+C(n) \cdot \underbrace{d}_{\text {Destruction }} \cdot \underbrace{e^{-p \cdot \Delta t}}_{\text {Outflow }} .
$$

Here, $\Delta t$ denotes the time between two pulses, $d$ gives the amount of contrast agent that is not destructed by the pulse $(0 \leq d \leq 1)$, and $p$ is the perfusion coefficient so that $e^{-p * d t}$ is the fraction of blood that is exchanged. Since the acquisition time is below 2 seconds, $p$ is assumed to be constant. The process is illustrated in Fig. 2, To calculate the concentration in the $n^{\text {th }}$ step directly, the following closed form exists:

$$
\begin{aligned}
C(n) & =C(1) \cdot\left(x^{n-1}+y \cdot \frac{x^{n-1}-1}{x-1}\right), \text { with } \\
x & =d \cdot e^{-p \cdot \Delta t} \quad \text { and } \quad y=1-e^{-p \cdot \Delta t} .
\end{aligned}
$$




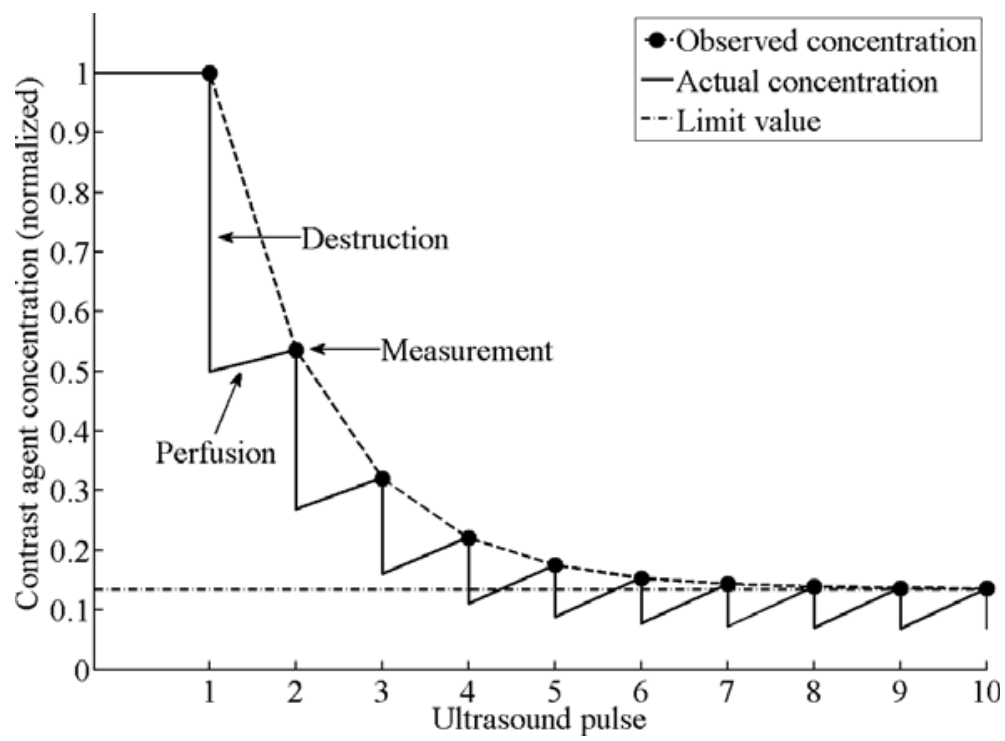

Fig. 2. Illustration of the DHI process with observed and actual concentration

The state of equilibrium between inflow, outflow and destruction that is eventually reached results in a constant intensity $I_{\infty}$ :

$$
\begin{aligned}
& I_{\infty}:=\lim _{n \rightarrow \infty} I(n) \stackrel{11}{=} \lim _{n \rightarrow \infty} I_{c}(n)+I_{b} \stackrel{2}{=} \lim _{n \rightarrow \infty} k \cdot C(n)+I_{b} \\
& \stackrel{\text { (4) }}{=} k \cdot C(1)\left[\lim _{n \rightarrow \infty} x^{n-1}+\lim _{n \rightarrow \infty} y \cdot \frac{x^{n-1}-1}{x-1}\right]+I_{b} \\
& \stackrel{p \geq 0}{=} I_{c}(1) \cdot \frac{-y}{x-1}+I_{b}=I_{c}(1) \cdot \frac{e^{-p \cdot \Delta t}-1}{d \cdot e^{-p \cdot \Delta t}-1}+I_{b} .
\end{aligned}
$$

Equation [5] shows that the relation between $I_{c}(n)$ and $C(n)$ does not have to be explicitly calculated. After solving equation 5 for $p$ we have:

$$
\begin{array}{ll}
p & =\frac{1}{\Delta t} \ln \frac{I_{c \infty} d-I_{c}(1)}{I_{c \infty}-I_{c}(1)} \quad \text { with } I_{c \infty}:=\lim _{n \rightarrow \infty} I_{c}(n) \\
& \stackrel{11}{=} \frac{1}{\Delta t} \ln \frac{I_{\infty} d-I(1)+I_{b}(1-d)}{I_{\infty}-I(1)}
\end{array}
$$

In this equation, $I(1)$ and $I_{\infty}$ can be taken from the US image series. The baseline intensity $I_{b}$ and the destruction coefficient $d$ are still missing. For this, we develop the following limit:

$$
\begin{aligned}
L_{n} & :=\lim _{\Delta t \rightarrow 0} \frac{I(n+1)-I_{b}}{I(n)-I_{b}} \lim _{\Delta t \rightarrow 0} \frac{I_{c}(n+1)}{I_{c}(n)} \\
& =\lim _{\Delta t \rightarrow 0} \frac{k C(1)\left[x^{n}+y \frac{x^{n}-1}{x-1}\right]}{k C(1)\left[x^{n-1}+y \frac{x^{n-1}-1}{x-1}\right]}=\frac{d^{n}}{d^{n-1}}=d
\end{aligned}
$$


Since equation 7 is valid for all $n$, we can equalize two arbitrary $L_{n}$ to solve this equation for $I_{b}$. We select $n=1$ and $n=2$ since at these frames flow effects are relatively small while the absolute destruction value is largest:

$$
\begin{aligned}
L_{1} & =L_{2} \\
\frac{I(2)-I_{b}}{I(1)-I_{b}} & =\frac{I(3)-I_{b}}{I(2)-I_{b}} \\
I_{b} & =\frac{I(2)^{2}-I(3) I(1)}{2 I(2)-I(3)-I(1)}
\end{aligned}
$$

Finally, we can obtain all values from the US image series and calculate $I_{b}$. Using equation 7 , we obtain $d$ also. To correct for possible errors due to image noise, we set $I_{b}$ to the highest value (255 with 8 bit grayscale images) when the denominator in equation 8 is zero and also constrain $I_{b}$ to the minimum of the time-intensity-curve.

\section{$3 \quad$ Results}

To validate our new method, we performed experiments with a flow phantom as well as examinations on humans to determine the brain perfusion.

\subsection{Phantom Experiments}

We designed a simple open-circuit flow model with a dialysis cartridge as a capillary phantom. The cartridge consisted of 9000 capillaries with an internal diameter of $200 \mu \mathrm{m}$. The fluid (nondegassed water at $22^{\circ} \mathrm{C}$ ) was driven by a programmable pump, which was connected to a roller pump head, providing continuous nonpulsatile flow at defined flow volumes. To avoid flotation of the UCA in the capillary-free entry section of the cartridge, it was fixed in a vertical position. The UCA was applied using a perfusion pump.
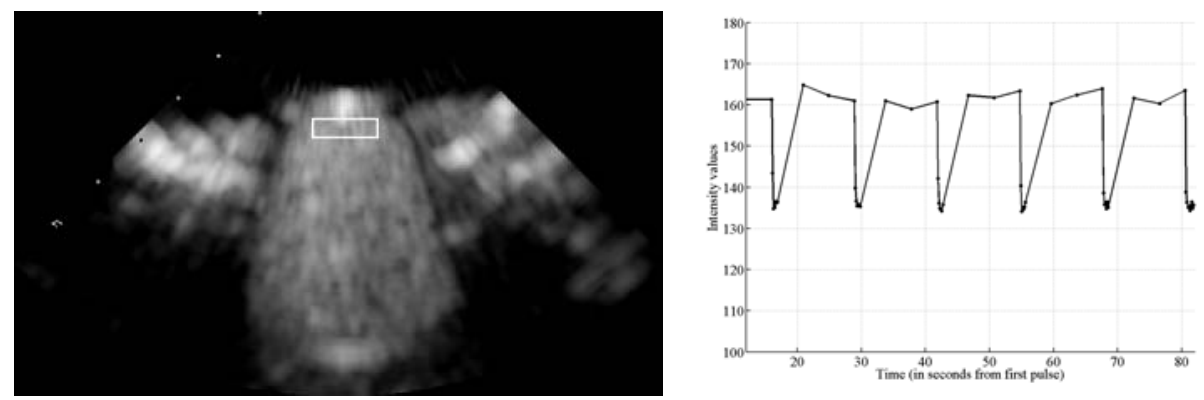

Fig. 3. Placement of the ROI (left) and exemplary time-intensity-curve (right) for the phantom experiment 

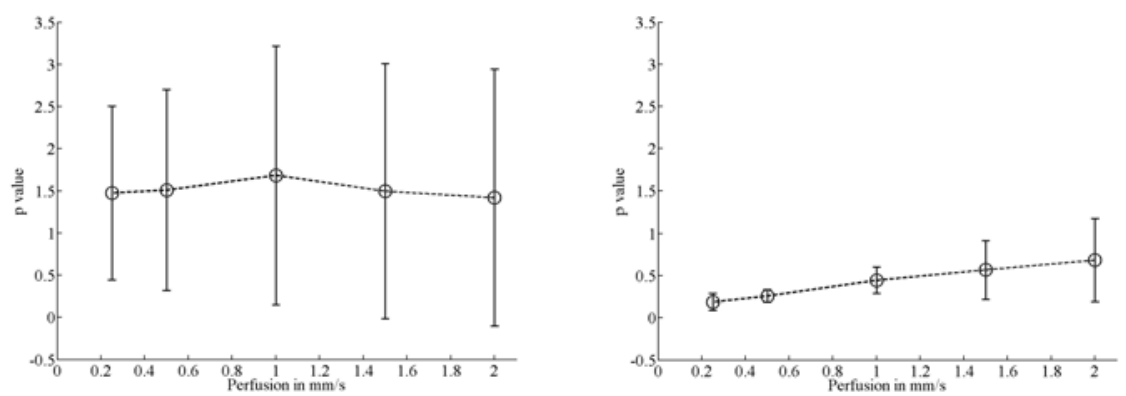

Fig. 4. Existing (left) and new (right) perfusion coefficient plotted over the known perfusion velocity

Using a custom-made probe holder, the US probe was held in place at an insonation angle of $90^{\circ}$ to the phantom, providing a cross-sectional view. Harmonic imaging was performed with a SONOS 5500 US system (Philips Medical Systems, Best, The Netherlands) and a 1.8-3.6 MHz sector transducer (S4 probe, Philips) in the conventional harmonic imaging mode (T-INT, insonation at 1.8 $\mathrm{MHz}$, receiver tuned to $3.6 \mathrm{MHz}$ ). The mechanical index (MI) was 1.6. Gain and transmit power settings were kept constant during the session. Standardized insonation conditions were provided by an acoustic stand-off.

Five flow velocities $(0.25,0.5,1,1.5$, and $2 \mathrm{~mm} / \mathrm{s})$ were investigated at five frame rates $(1,1.33,2,4$ and $6.67 \mathrm{~Hz})$. The rapid, destructive US sequences were followed by three pulses at $0.25 \mathrm{~Hz}$ to allow replenishment of intact microbubbles into the insonation plane. To assess the repeatability of the measurements, each combination was repeated 6 times during one single session.

To exclude attenuation phenomena that occur in deeper regions of the flow model, we analyzed contrast data of a rectangular region of interest (ROI), which was placed at a distance of $30 \mathrm{~mm}$ from the probe. Fig. 3 shows the placement of the ROI as well as an exemplary time-intensity curve of one sequence.

The obtained images were used to calculate $p$ in the ROI according to the model given in [6] and for our new method. Since in the phantom experiment we have the perfusion ground truth available, we put the calculated $p$ values in relation to the known perfusion velocities. The most impressive results are shown in Fig. 4. It is clearly visible that the new perfusion coefficient correlates highly to the perfusion velocity which is not the case for the existing model.

The appearance of the new perfusion coefficient also strongly supports the theory of a linear relationship. Fitting a line onto the obtained values of $p$ gives

$$
P=3.47 \cdot p+0.44
$$

with $P$ being the known perfusion velocity.

\subsection{Brain Perfusion}

Among applications of perfusion analysis, determining brain perfusion with transcranial sonography is considered most challenging, since US images suffer from 

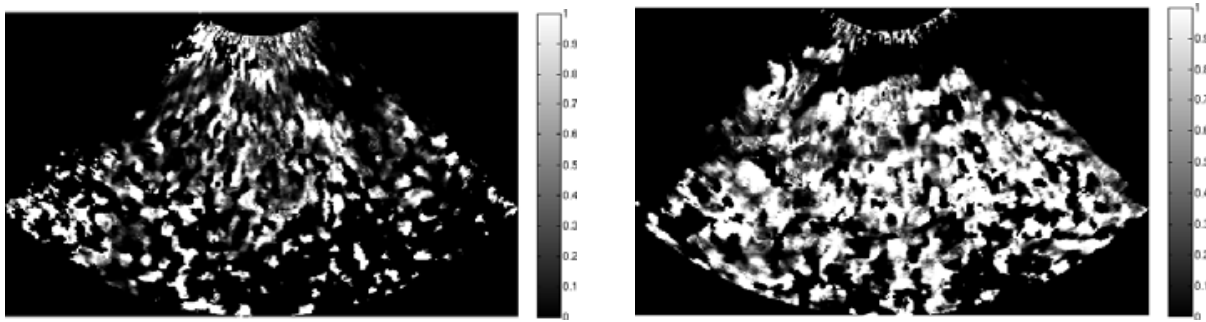

Fig. 5. Visualizations of the perfusion coefficient for a healthy subject (left) and a patient suffering from an ischemic stroke (right)

a low SNR due to the high impedance of the skull. When acquiring a DHI sequence, the penetration depth of the ultrasound beam is set to $10 \mathrm{~cm}$. Thus, two sequences are necessary to cover the diencephalic imaging plane from both hemispheres of a patient.

Fig. 5 shows two visualizations of the perfusion coefficient for two different subjects. Both sequences have been acquired in an axial imaging plane with the US probe at the temporal acoustic bone window at a frame rate of $6.67 \mathrm{~Hz}$. Values of $p$ were calculated with the new method and visualized as gray-level images. The most interesting part of the brain regarding pathological brain perfusion is close to the skull, i.e. close to the top in the US frame, because (a) most perfusion deficits occur in this area and (b) the lower part of the US image area increasingly suffers from the UCA attenuation or shadowing effect and hence can be ignored in the further analysis.

The images may be difficult to grasp, but in comparison it becomes evident that a large region to the top of the right image, i.e. at the cortex of the ischemic stroke patient, has a perfusion coefficient value of zero. That means this region is not perfused at all which was also confirmed by an MRI examination of this patient.

This visualization is of course only semi-quantitative and does not incorporate the findings from Section 3.1. A fully quantitative mapping using the relationship given in equation 9 is not feasible, since many influencing factors hinder the comparison of the phantom experiment with the human examination.

\section{Discussion and Conclusions}

Compared to currently used diagnostic procedures the presented method features fast and inexpensive perfusion analysis by means of ultrasound image sequences. An examination can be performed directly at the patient's bedside under minimal strain for the patient. Especially noteworthy is the short examination time of the DHI method compared to other US imaging methods. Besides further stress reduction for the patient it helps in providing stable insonation conditions and thus makes it the most promising method among its competitors.

Our model extension greatly enhances prediction accuracy for the perfusion coefficient and supports the theory of a linear relation to the underlying perfusion 
as shown in the in vitro experiments. Most interesting is the fact that the standard deviation of the perfusion coefficient is smaller the lower the perfusion is. These regions of malperfused, i.e. ischemic, tissue are of high diagnostic interest and a high accuracy is especially important.

Although setup to mimic human microcirculation conditions, transfering the findings of the in vitro to the in vivo case is still a challenging task, since many factors influence the relationship determined in equation 9. Besides imaging parameters, such as mechanical index, UCA-related parameters have to be considered, i.e. the type of UCA, its concentration etc. Furthermore, patient-related paramters exist, like the accessibility of the acoustic bone window. However, our findings are a great step towards fully quantitative perfusion analysis of microcirculation with ultrasound. Still, comprehensive phantom studies are needed to provide a basis to cover all parameter combinations.

To summarize, our method is very promising. The ultrasound-based diminution harmonic imaging method provides a fast and inexpensive alternative among different modalities for microcirculation evaluation. Because of the general approach it is not restricted to cerebral microcirculation and can be applied to every organ that is reachable by ultrasound.

\section{References}

1. Hancock, J., Dittrich, H., Jewitt, D., Monagham, M.: Evaluation of myocardial, hepatic, and renal perfusion in a variety of clinical conditions using an intravenous ultrasoundcontrast agent (optison) and second harmonic imaging. Heart 81(6), 636641 (1999)

2. Seidel, G., Meyer, K.: Impact of ultrasound contrast agents in cerebrovascular diagnostics. European Journal of Ultrasound 16(1-2), 81-90 (2002)

3. Krogias, C., Postert, T., Meves, S., Wilkening, W., Przuntek, H., Eyding, J.: Semiquantitative analysis of ultrasonic cerebral perfusion imaging. Ultrasound in Medicine \& Biology 31(8), 1007-1012 (2005)

4. Maciak, A., Kier, C., Seidel, G., Meyer-Wiethe, K., Hofmann, U.G.: Detecting stripe artifacts in ultrasound images. Journal of Digital Imaging (2007)

5. Wilkening, W., Postert, T., Federlein, J., Kono, Y., Mattrey, R., Ermert, H.: Ultrasonic assessment of perfusion conditions in the brain and in the liver. In: Proc. IEEE Ultrasonics Symposium, San Juan, Puerto Rico, October 2000, vol. 2, pp. 1545-1548 (2000)

6. Kier, C., Meyer-Wiethe, K., Seidel, G., Aach, T.: Ultrasound cerebral perfusion analysis based on a mathematical model for diminution harmonic imaging. Methods of Information in Medicine 46(3), 308-313 (2007)

7. Williams, Q.R., Noble, J.A.: A spatio-temporal analysis of contrast ultrasound image sequences for assessment of tissue perfusion. In: Barillot, C., Haynor, D.R., Hellier, P. (eds.) MICCAI 2004. LNCS, vol. 3217, pp. 899-906. Springer, Heidelberg (2004) 